\title{
Differential effect of body mass index on the incidence of diabetes and diabetic retinopathy in two Asian populations
}

Joel Chee Yee Chan', Miao Li Chee', Nicholas Yi Qiang Tan ${ }^{1,2,3}$, Ching-Yu Cheng ${ }^{1,2,3}$, Tien Yin Wong ${ }^{1,2,3}$ and Charumathi Sabanayagam (1) 1,2,4

\begin{abstract}
Aims: To examine the association of body mass index (BMI) with the incidence of diabetes mellitus (DM) and diabetic retinopathy $(D R)$ in Asians.

Methods: We analysed data from 4101 adults (Malay, $n=1901$ and Indian, $n=2200$ ) who participated in the baseline (2004-2009) and 6-year follow-up (2011-2015) of two independent population-based studies with similar methodology in Singapore. BMl was categorised into normal $\left(<25 \mathrm{~kg} / \mathrm{m}^{2}\right)$, overweight $\left(25-29.9 \mathrm{~kg} / \mathrm{m}^{2}\right)$ and obese $\left(\geq 30 \mathrm{~kg} / \mathrm{m}^{2}\right.$ ). DM was diagnosed as random plasma glucose $\geq 200 \mathrm{mg} / \mathrm{dL}, \mathrm{HbA} 1 \mathrm{c} \geq 6.5 \%$ or self-reported physician diagnosed DM. DR was assessed from retinal photographs graded using a standard protocol. The associations of baseline BMI with incident DM and DR was examined using multivariable poisson regression models adjusting for potential confounders including duration of $\mathrm{DM}$, family history of $\mathrm{DM}$ and $\mathrm{HbA}_{1 \mathrm{c}}$.

Results: The incidence of DM was $12.8 \%$ and among 1586 participants with DM, the incidence of DR was $17.6 \%$ over a median follow-up period of 6.2 years. Compared to those with $\mathrm{BMI}<25$, the relative risk ( $95 \%$ confidence interval) of incident DM was 1.77 (1.36-2.29) for overweight and 2.01 (1.50-2.71) for obese ( $p$ trend $<0.001)$. Relative risk of DR was $0.80(0.59-1.09)$ for overweight and $0.60(0.39-0.92)$ for obese $(p$ trend $=0.02)$. In analyses stratified by ethnicity, similar pattern of associations with DM and DR were observed in both ethnicities.
\end{abstract}

Conclusion: Our results suggest that, overweight and obesity increased the 6-year risk of DM but decreased the 6-year risk of DR in these Asian populations.

\section{Introduction}

Diabetes mellitus (DM) is projected to increase to 592 million in 2035, with Asia having the highest number of individuals with DM globally and importantly, with the prevalence increasing at a much faster rate than in

\footnotetext{
Correspondence: Charumathi Sabanayagam (charumathi.sabanayagam@seri. com.sg)

${ }^{1}$ Singapore Eye Research Institute, Singapore National Eye Centre, The Academia, 20 College Road, Discovery Tower Level 6, Singapore 169856, Singapore

${ }^{2}$ Department of Ophthalmology, Yong Loo Lin School of Medicine, National University of Singapore, Singapore, Singapore

Full list of author information is available at the end of the article
}

Western countries ${ }^{1}$. Diabetic retinopathy (DR), a major complication of DM, accounts for $5 \%$ of all blindness affecting up to 2 million people worldwide yet up to $90 \%$ of blindness resulting from DR is preventable if adequate screening and evidence-based care for DM were to be implemented $^{2}$. The classic risk factors for DR include longer duration of $\mathrm{DM}$, poor glycemic control reflected by higher glycated haemoglobin $\left(\mathrm{HbA}_{1 \mathrm{c}}\right)$ levels and hypertension $^{3-6}$.

While obesity is an established risk factor for $\mathrm{DM}^{7}$, and has been postulated to be also a risk factor for the development of $\mathrm{DR}^{8}$, the association of body mass index 
(BMI) with DR in previous studies have not shown consistent results. Furthermore, Asians have been shown to have different associations between BMI, percentage body fats and health risks such as cardiovascular disease as compared to Western populations ${ }^{9-11}$. Previous research suggests that BMI could influence DR differently in Asian and Western populations ${ }^{8,} 12-20$. Thus, while Western studies have showed higher BMI and obesity is related to $\mathrm{DR}^{8,11,12}$ the majority of studies in Asia have shown that lower $\mathrm{BMI}$ is related to $\mathrm{DR}^{13-17,}{ }^{19}$. Importantly, few studies have been prospective in nature ${ }^{10,11}$ and the relationship between baseline BMI and the subsequent risk of DR is unclear.

To address these gaps, in two we examined the relationship of baseline BMI with 6-year risk of DM and the 6-year risk of DR in Asian adults with DM in Singapore.

\section{Methods}

\section{Study participants}

Data for the current study was derived from two prospective cohort studies: the Singapore Malay Eye Study (SiMES, 2004-2006, SiMES-2, 2011-2013), and the Singapore Indian Eye Study (SINDI, 2007-2009, SINDI-2, 2012-2015). Details of the design of the above studies, sampling plan and methodology have been extensively published elsewhere ${ }^{21,22}$.

In brief, a total of 3280 of the 4168 eligible individuals participated at baseline in SiMES (78.7\% response rate), and 3400 of the 4497 eligible individuals participated at baseline in SINDI (75.6\% response rate). Of the 3280 participants in the SiMES cohort, 1052 had DM and 295 had DR at baseline; of the 3400 participants in the SINDI cohort, 1320 had DM and 418 had DR at baseline. In the respective follow-up studies (median follow-up period of 6.2 years), a participant from the original studies was considered to be subsequently ineligible if the he or she had moved from the address of residence, had not lived in the residence in the past 6 months, or was terminally ill or deceased. Thus, after excluding 644 and 486 ineligible SiMES and SINDI participants respectively, 1901 of the 2636 (72.1\% response rate) eligible SiMES participants, and 2200 of the 2914 (75.5\% response rate) eligible SINDI participants attended the follow-up studies ${ }^{23,24}$.

For the current analyses, we included only participants who attended both baseline and follow-up (1901 Malays and 2200 Indians). Since the methodology for SiMES and SINDI were similar, we combined data from the two cohorts for the main analysis. For outcome, 'Incidence of DM', we excluded 2372 participants with prevalent DM at baseline, and 4 participants with missing data on key variables; this left us with 2403 participants without DM for inclusion (Fig. 1). For outcome, 'Incidence of DR', we excluded 713 participants with prevalent DR at baseline, and 2 participants with missing data; this left us with 908 participants with DM eligible for inclusion in the analysis (Fig. 1). For outcome, 'Incident DM', our sample size of 2403 and 308 number of cases had $80 \%$ power to detect a RR of minimum 1.55 comparing normal versus overweight and 1.76 comparing normal versus obese respectively. For continuous BMI, we had $80 \%$ power to detect RR of minimum 1.03 for incident DM per unit increase in BMI. For outcome, 'Incident DR, our sample size of 908 and 160 number of cases had $80 \%$ power to detect a RR of minimum 0.67 comparing normal versus overweight and 0.63 comparing normal versus obese respectively. For continuous BMI, we had $80 \%$ power to detect RR of

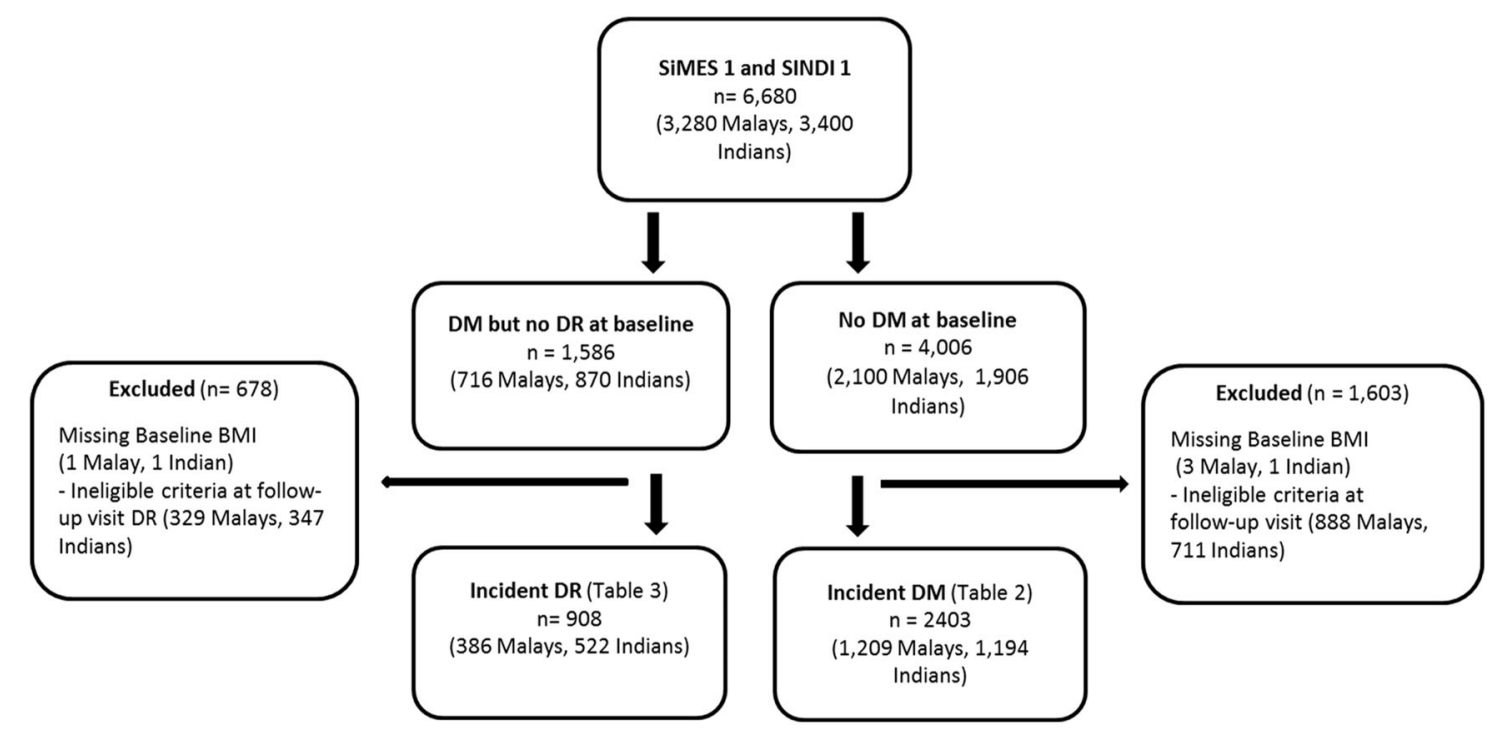

Fig. 1 Flowchart for the inclusion and exclusion of participants 
minimum 0.93 for incident DR per unit increase in BMI. The above power calculations were done based on significance level of 0.05 .

The study adhered to the Declaration of Helsinki, ethics approval was obtained from the SingHealth Centralised Institutional Review Board, and written informed consent was obtained from all participants.

\section{Assessment and definition of DM, BMI and other risk factors}

All participants underwent a standardised questionnaire, clinical examination and the collection of blood samples to assess for non-fasting glucose, $\mathrm{HbA}_{1 \mathrm{c}}$ and lipid profile. Questionnaire data included ethnicity, education, income, personal history of DM, hypertension, family history of DM, smoking history, use of medications and duration of DM. Income level was categorised into two groups (<Singapore dollar $[S \$ 2000$, and $\geq S \$ 2000$ ); level of education was also categorised into two groups (primary school education and below and above primary school education).

Height was measured in centimetres using a wallmounted measuring tape and weight was measured in kilograms using a digital scale (SECA, model 7,822,321,009; Vogel and Halke, Germany). BMI was defined as the weight in kilograms divided by the square of height in metres $\left(\mathrm{kg} / \mathrm{m}^{2}\right)$. BMI was categorised into underweight $\left(\mathrm{BMI}<18.5 \mathrm{~kg} / \mathrm{m}^{2}\right)$, normal $(18.5 \leq \mathrm{BMI}<$ $\left.25 \mathrm{~kg} / \mathrm{m}^{2}\right)$, overweight $\left(25 \leq \mathrm{BMI}<30 \mathrm{~kg} / \mathrm{m}^{2}\right)$ and obese $\left(\geq 30 \mathrm{~kg} / \mathrm{m}^{2}\right)$ according to BMI cut-off points ${ }^{9}$. BP measurements were taken using a digital automatic blood pressure monitor after the participant was seated for at least $5 \mathrm{~min}$ and an average of two measurements were taken as the blood pressure value for that individual. Hypertension was defined as a systolic BP of $140 \mathrm{mmHg}$ or greater, diastolic BP of $90 \mathrm{mmHg}$ or greater, or use of antihypertensive medication ${ }^{25}$. A standardised questionnaire was conducted for all participants in English or their mother tongue (Malay or Tamil) by trained interviewers. DM was defined as self-reported physician's diagnosis, use of insulin, use of oral hypoglycaemia medications or random plasma glucose $\geq 200 \mathrm{mg} / \mathrm{dL}$ or $\mathrm{HbA}_{1 \mathrm{c}} \geq 6.5 \%(48 \mathrm{mmol} / \mathrm{mol})^{26,27}$. We defined type $1 \mathrm{DM}$ as those who developed DM before the age of 30 years. For the incident analysis, since we excluded those with DM including those who developed DM before the age of 30 years $(n=8)$, we assume that those who developed DM at follow-up fall under type $2 \mathrm{DM}$, i.e., age of onset after 30 years. Incident DM was defined as onset of DM at the follow-up among those who were free of DM at baseline.

Retinal photography and diabetic retinopathy assessment

Two-field retinal photography was taken after pupil dilation according to the Early Treatment for DR Study
(ETDRS) protocol using a $45^{\circ}$ nonmydriatic digital retinal camera (Canon CR-DGI with a 10D/20D SLR backing, Canon, Japan) ${ }^{27}$. Retinal images were graded for DR by trained graders masked to participant characteristics. Lesions considered to be characteristic of DR were: microaneurysms, haemorrhages, cotton wool spots, intraretinal microvascular abnormalities, hard exudates, venous beading and new vessels were seen ${ }^{28}$. For each eye, DR severity score was assigned based on the modified Airlie House Classification ${ }^{28}$ into none (ETDRS levels 10-15), minimal non-proliferative (NPDR, level 15-20), mild NPDR (level 35), moderate NPDR (level 43-47), severe NPDR (level 53), or proliferative DR (PDR, score $>60)^{28}$. Based on the worse eye score, any DR was defined as a severity level of 15 and above and vision-threatening DR (VTDR) as presence of severe NPDR, PDR, or clinically significant diabetic macular edema $(\mathrm{DME})^{28}$.

Incident DR was defined as severity level $>15$ and incident VTDR as those who developed VTDR at the follow-up visit among those who were free of VTDR at baseline (which includes minimal, mild, moderate NPDR).

\section{Statistical analysis}

Analyses were conducted using Stata Version 14.0 (StataCorp LP, College Station, TX) separately for the two main outcomes: incident DM and incident DR. For the present analysis, since the prevalence of being underweight was low (3.1\%), we combined underweight and normal as one category and defined normal as $\mathrm{BMI}<25$ $\mathrm{kg} / \mathrm{m}^{2}$. We compared baseline characteristics of participants stratified by those 1) with and without DM and 2) with and without DR using the Chi-square test or the independent $t$-test as appropriate for the variable. We examined the associations of baseline BMI (categorical, as well as continuous, per SD increment) with the two outcomes using two poisson regression models with robust variance: ${ }^{29}$ 1) an age, gender and ethnicity-adjusted model; 2) a multivariable model adjusting for other covariates as well, such as income and education, current smoking, family history of $\mathrm{DM}, \mathrm{HbA}_{1 \mathrm{c}}$, total cholesterol, HDL cholesterol, systolic BP and diabetes duration. Ptrend was calculated using BMI categories as an ordinal variable. To examine for consistency of the association between BMI and incident DM or incident DR, we performed subgroup analyses stratified by gender and ethnicity. We examined interactions by gender and ethnicity by including cross-product interaction terms in the corresponding multivariable models. Additionally, the associations of BMI and incident VTDR was also examined with multivariable poisson regression models with robust variance $^{29}$. In supplementary analyses, firstly, we excluded underweight participants $(n=84)$ from the analyses and repeated the main multivariable models; secondly, we examined the associations using WHO Asian BMI 


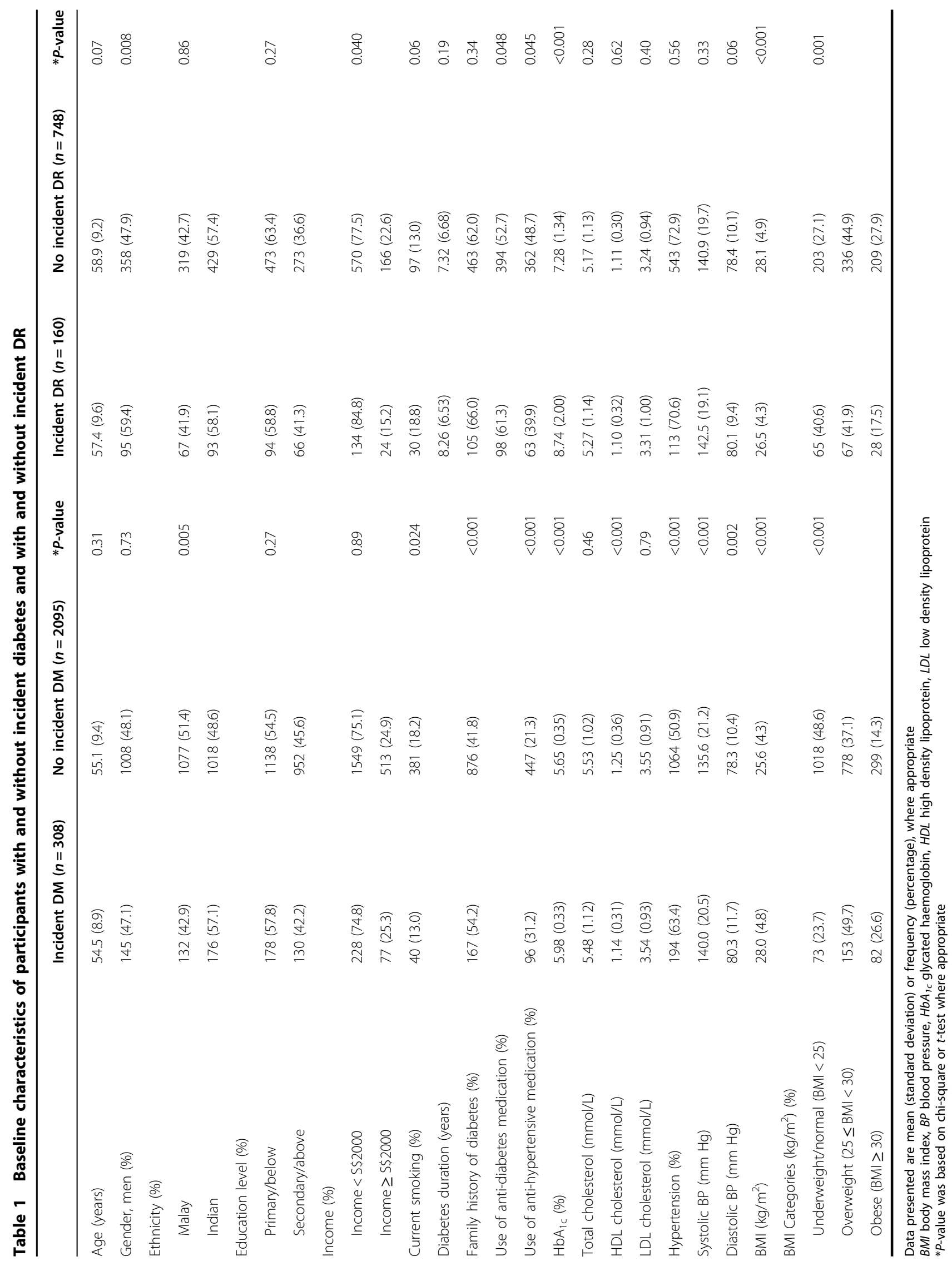




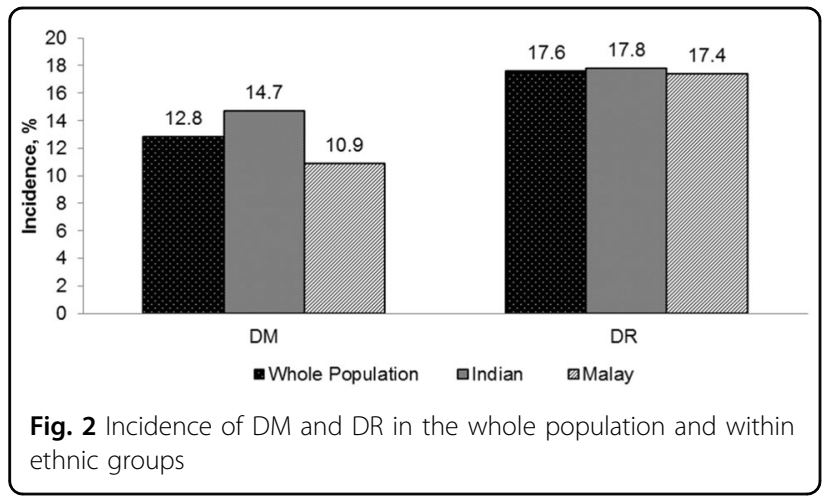

categories (BMI $<23,23-27.5, \geq 27.5$ for normal, overweight and obese) $)^{9}$. In the current study, since only 33 participants (3.7\%) were under treatment with fenofibrate and 16 participants were on insulin $(1.8 \%)$, we did not assess the influence of these drugs in the onset of DR.

\section{Results}

Baseline characteristics of the study population are presented in Table 1. Compared to participants with no DM, those with DM were more likely to be Indians, and less likely to be current smokers; had higher prevalence of hypertension and use of anti-hypertensive medications, family history of $\mathrm{DM}$; had higher levels of $\mathrm{HbA}_{1 \mathrm{c}}$, $\mathrm{BMI}$, systolic and diastolic BP and lower HDL cholesterol level. Compared to participants with no DR, those with DR were younger, more likely to be men and current smokers, had higher prevalence of antidiabetic medication use, higher levels of $\mathrm{HbA}_{1 \mathrm{c}}$, diastolic $\mathrm{BP}$ and total cholesterol but lower prevalence of anti-hypertensive medication use and had lower levels of income and BMI.

Over a median follow-up period of 6.2 years, incidence of DM was $12.8 \%$ in the overall population, and 10.9 and $14.7 \%$ in the Malay and Indian populations respectively $(p$ $=0.001$ ); incidence of DR was $17.6 \%$ in the overall population, and 17.4 and $17.8 \%$ in the Malay and Indian populations respectively (Fig. 2). There was a significant difference in the incidence of DM between Indians and Malays but no significant difference was observed with regards to incidence of DR.

Table 2 shows the associations between baseline BMI and incident DM. The incidence of DM was significantly higher in overweight and obese subjects than in subjects with $B M I<25 \mathrm{~kg} / \mathrm{m}^{2}$. The RR of incident DM was higher in both the overweight (1.77) and obese (2.01) groups and also per SD increase in BMI in multivariable analysis after adjusting for potential confounders. In stratified analysis, the positive associations between overweight, obesity and incident DM remained significant across ethnic and gender subgroups without any significant interactions $(P$ values of the interaction terms of $\mathrm{BMI}^{*}$ gender and BMI*ethnicity were 0.87 and 0.15 respectively).
Table 3 shows the associations between BMI and incident DR among persons with DM at baseline. The incidence of DR in the overweight and obese subjects was significantly lower than in subjects with $\mathrm{BMI}<25 \mathrm{~kg} / \mathrm{m}^{2}$. Obesity was significantly associated with DR in both age, sex, ethnicity-adjusted and the multivariable models. Overweight, while associated with DR in the first model, the association lost significance in the multivariable model. The inverse association between BMI and incident DR persisted when BMI was analysed as a continuous variable. In subgroup analysis by gender, the association between higher BMI and lower risk of DR was significant in women; in men, although the association was no longer statistically significant, the association remained consistent in direction (inverse). After stratification by ethnicity, in both Malay and Indian groups, the associations between higher BMI (as a categorical or continuous variable) and lower risk of incident DR remained consistent in direction, although no longer statistically significant. In analyses using incident VTDR as an outcome $(n=50)$, no significant associations were observed between BMI (as either a categorical or continuous variable) and VTDR (Table 4).

In supplementary analyses, we repeated the analyses in Tables 2 and 3 1) excluding underweight participants ( $n$ $=79$ and 5 respectively), 2) using BMI cut-points for public health action in Asians, recommended by $\mathrm{WHO}^{9}$. In these two independent analyses, the associations between BMI (as a continuous or as categorical variables) and incident DM remained statistically significant and consistent similar to the main analyses in Table 2. Furthermore, compared to normal, being underweight did not significantly affect the risk of incident DM or DR.

\section{Discussion}

In this population-based cohort of Asian adults of Malay and Indian ethnicities, we showed that higher BMI was associated with an increased incidence of DM, but lower incidence of DR, independent of potential cofounders such as age, gender, ethnicity, duration of DM and $\mathrm{HbA}_{1 \mathrm{c}}$. These associations were consistent when BMI was analysed either as a categorical or continuous variable. In addition, the associations were consistent in analyses stratified by gender and ethnicity.

Our results for the positive association between increased BMI and incident DM are consistent with that of previous prospective studies, mostly in Western populations ${ }^{7,30}$. The reasons for the positive association between BMI and DM have been well described and include insulin resistance and progressive beta cell dysfunction ${ }^{31}$. Three mechanisms linking obesity to insulin resistance include: (1) increased production of cytokines, including tumour necrosis factor- $\alpha$, resistin and retinolbinding protein 4 , leading to reduced levels of adiponectin 


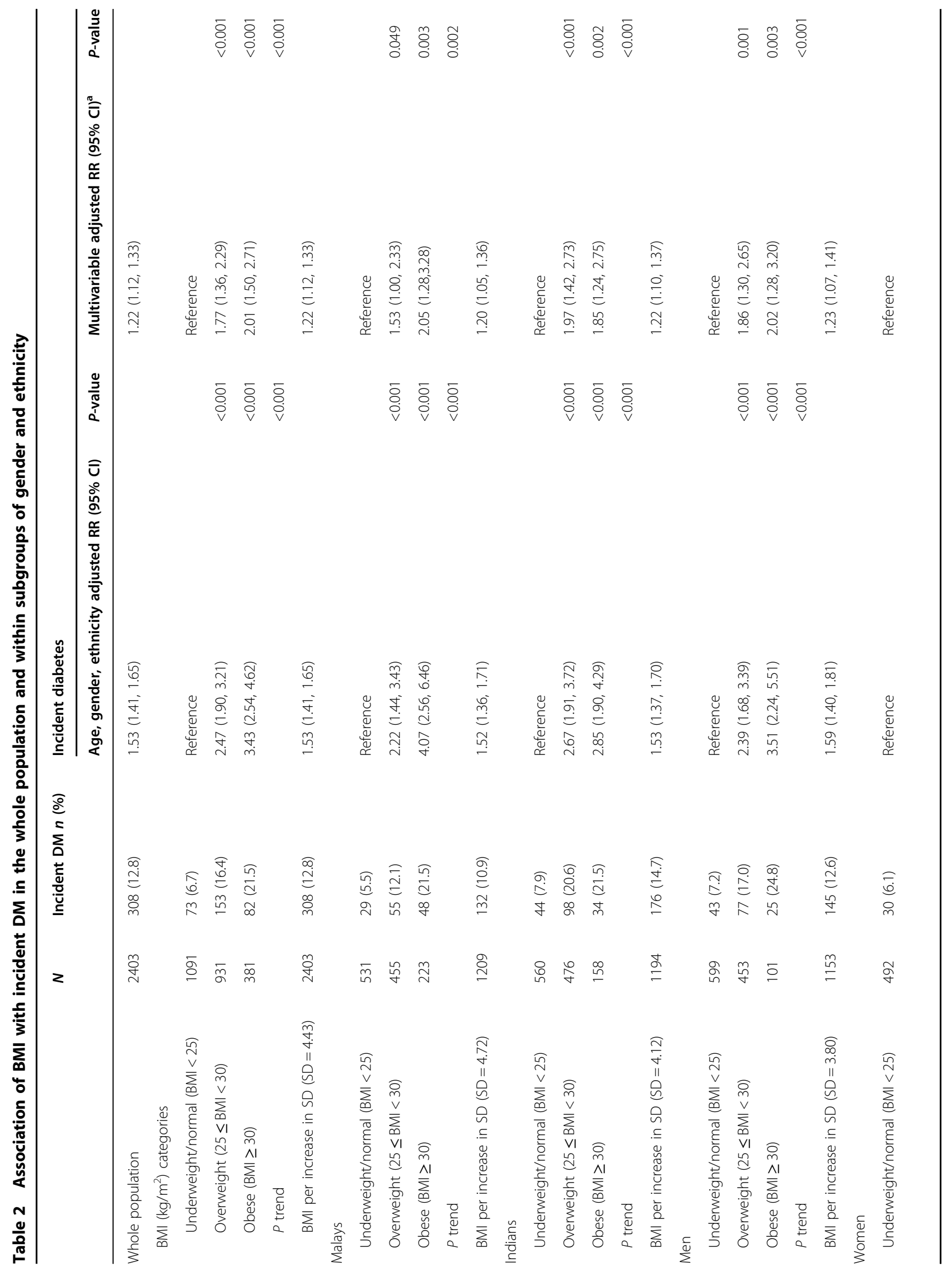




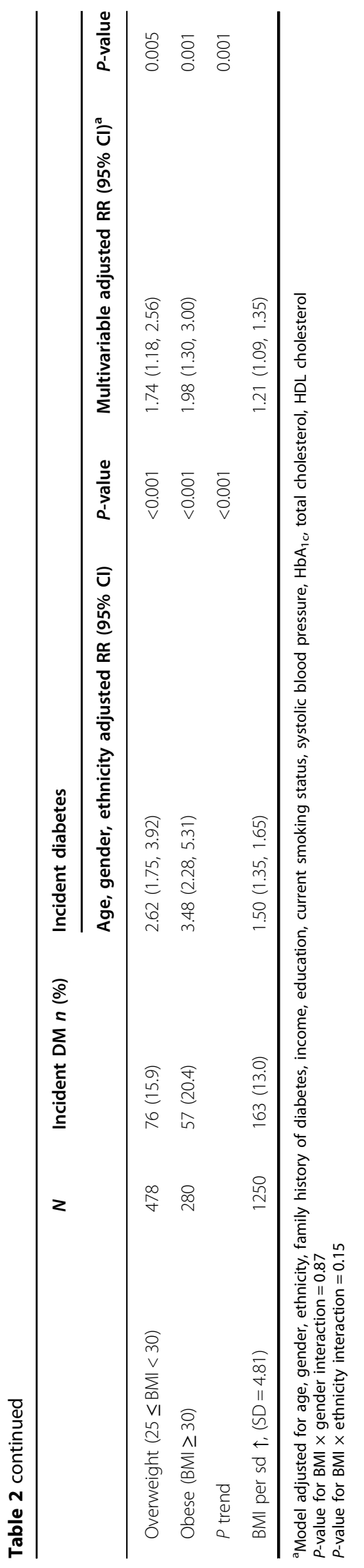

and subsequent insulin resistance; ${ }^{32}$ (2) ectopic fat deposition in the liver and in the skeletal muscle; ${ }^{33}$ and (3) mitochondrial dysfunction leading to decreased insulin sensitivity. Some studies have also hypothesised a similar underlying defect whereby obesity induced cellular damage activate macrophages which worsens tissue inflammation leading to the pathogenesis of insulin resistance in the liver and peripheral tissues and resulting in damage to the $\beta$-cells ${ }^{34,35}$. Hence, higher BMI can lead to an increased incidence of DM due to insulin resistance and $\beta$-cell dysfunction.

In the current study, we found an inverse association between obesity and incident DR and also between BMI (continuous) and DR. Our findings are similar to a number of cross-sectional Asian studies showing a protective association between overweight/obesity and $\mathrm{DR}^{13-17,19}$. For instance, in the Beijing Community Diabetic Study, the Chennai Urban Rural Epidemiology Study and in a study of Asian Indian, Chinese and Creole Mauritians, higher BMI was associated with lower prevalence of $D R$, with the reported OR ranging from 0.50 to 0.95 in these studies ${ }^{13}, 16,19$. Contrary to our study findings, most of the prospective studies done in Western populations had shown a positive association between higher $\mathrm{BMI}$ and $\mathrm{DR}^{8,12,20}$. For instance, the Diabetes Control and Complications Trial reported that higher BMI was significantly associated with incidence of DR (OR 1.11 (1.01-1.24)) among patients with type $1 \mathrm{DM}^{20}$. In another study in Australia, higher BMI was significantly associated with any DR (OR 1.06, 1.01-1.11) . To the best of our knowledge, there have been no studies examining the effect of BMI on the incidence of DR in Asian populations. Thus, it appears that the association of BMI and DR may differ between Asian and Western populations. In the current study, although overweight category showed a lower OR for incident DR similar to obesity category, the association lacked significance $(p=$ 0.16 ). This could be due to the smaller number of cases in the overweight category. However, the $p$-trend across categories was significant $(p$-trend $=0.02)$ and in analyses using BMI as a continuous variable, a similar significant inverse association was found.

The exact mechanisms underlying the inverse association between BMI and DR in these Asian populations are uncertain. It has been shown that for the same BMI, body fat among Asians is higher by $3-5 \%$ as compared to Westerners $^{36}$ in other words, for the same BMI, Asians and Westerners may have different levels of adiposity. However, even after adjusting for this by using the lower WHO Asian BMI cut-off values, our results still showed higher BMI was associated with lower incidences of DR. This inverse association ties in with the "obesity paradox", which describes the trend of higher BMI being associated with better outcomes after percutaneous coronary 


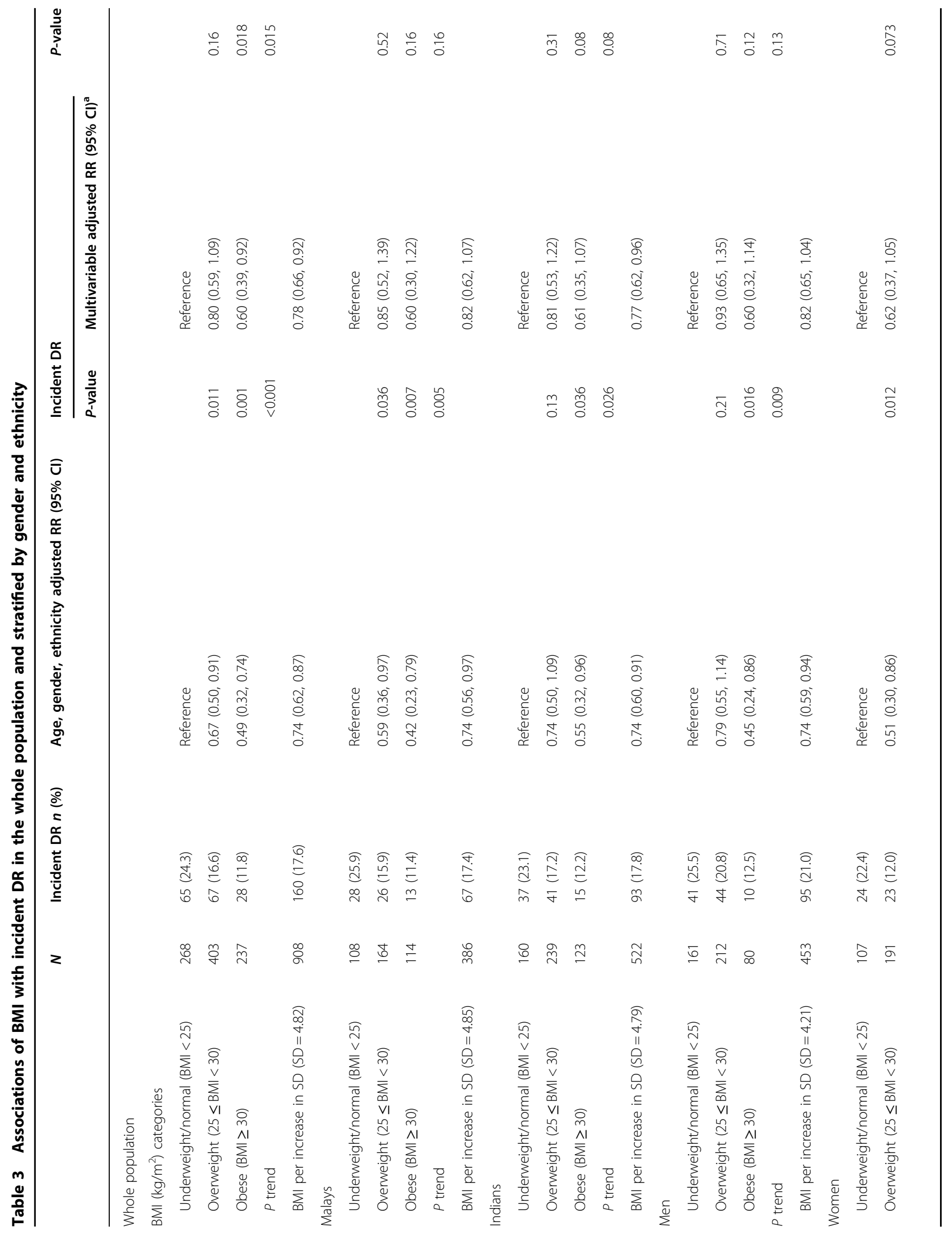



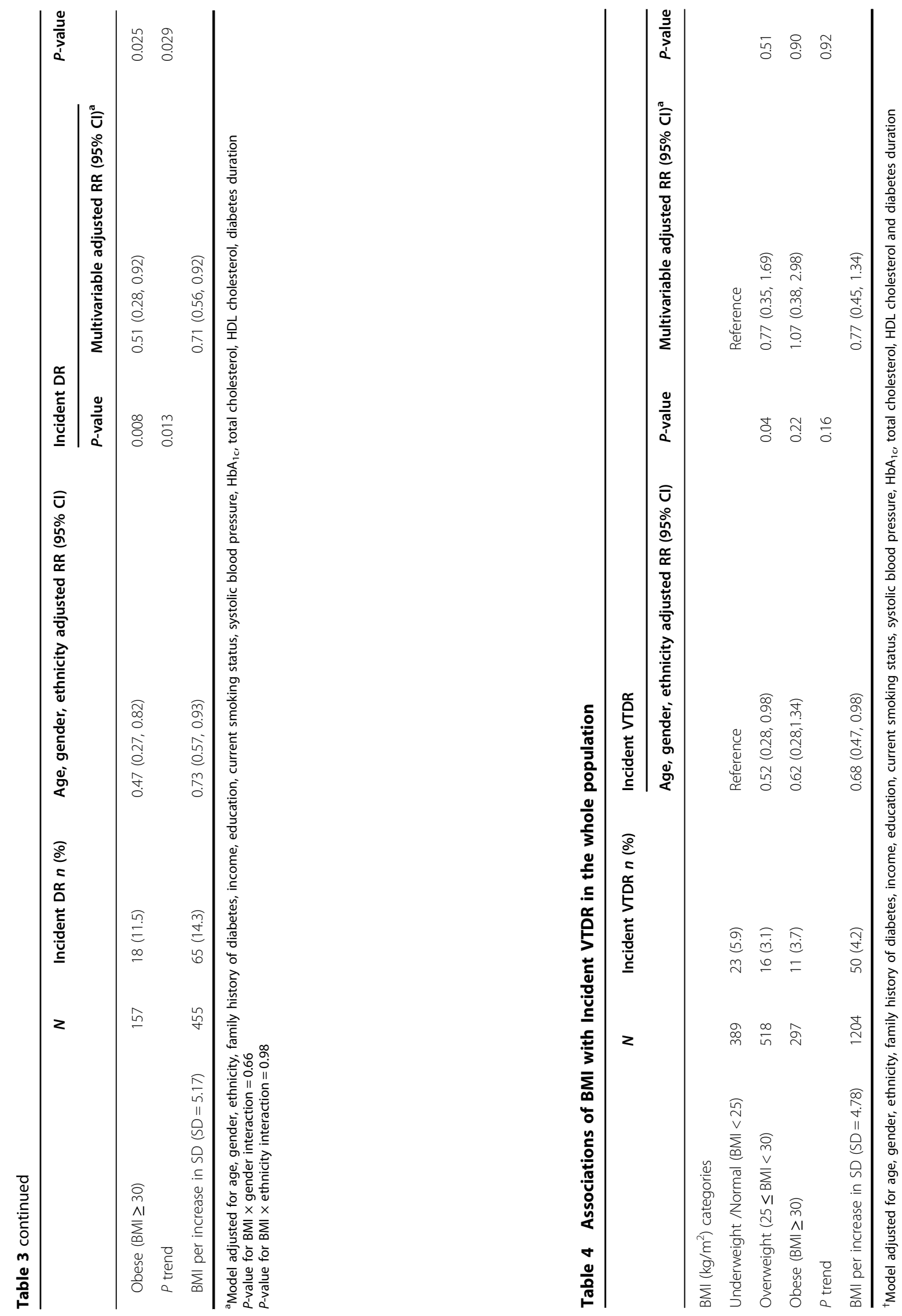
intervention, as well as in conditions such as chronic kidney disease, congestive cardiac failure, peripheral arterial disease, stroke risk and thromboembolism ${ }^{37-40}$. Alternatively, it may not be that higher BMI is protective towards DR, but that individuals with lower BMI may have more severe DM (as patients with decompensated disease may undergo a catabolic phase resulting in unintentional weight loss) and thus have a higher risk of developing DR. Generally, long-term diabetes duration is associated with a lesser capacity for insulin secretion and these participants tend to have a lower BMI as compared to participants with shorter diabetes duration ${ }^{41}$. This could also be an important factor explaining the inverse relationship between BMI and the incidence of DR.

Evidence linking BMI levels with VTDR, has thus far been inconclusive, with some studies showing higher risk of VTDR with lower $\mathrm{BMI}^{42}$, and others with higher $\mathrm{BMI}^{43}$. In our study, no significant association was found between BMI and the risk of developing VTDR despite the incidence of VTDR being lower in the overweight (3.1\%) and obese (3.7\%) groups compared to those with $\mathrm{BMI}<25$ (5.9\%). The reason for this is unclear, although our analyses were limited by a small number of cases of $\operatorname{VTDR}(n=50)$. Because of the smaller number of events, we were also unable to examine DME and PDR separately.

Despite our findings that higher BMI is associated with lower risk of developing DR, caution must be made not to over-generalise that a higher $\mathrm{BMI}$ is preferable. Consistent with results of this study, it has been well reported that higher BMI is associated with increased risk of developing $\mathrm{DM}^{7},{ }^{30}$. Furthermore, and despite various studies suggesting a protective role of higher BMI in certain disease states as per the "obesity paradox", it should be remembered that obesity has been associated with many health risks, as well as overall increased mortality ${ }^{44}$.

The strengths of this study include a large sample size of two different ethnicities; longitudinal data over a 6-year period with a relatively low drop-out rate; and a standardised and comprehensive protocol for grading DR and assessing risk factors. One of our limitations arises from BMI being an imperfect measurement of body fat and composition, with more accurate alternative measures such as waist hip ratio to measure central abdominal obesity having been described ${ }^{15}$. Furthermore, this being an epidemiological study involving a large population, we only had baseline and follow-up HbA1c measurements and hence were unable to use the mean of serial HbA1c measurement throughout the 6-year period. Additionally, we were not able to adjust for the effects of other potential confounders such as diet and physical activity as such data were not collected.

In conclusion, we showed in this large cohort of Asian Malays and Indians, that higher BMI was associated with a higher incidence of DM but a lower incidence of DR over a 6-year period. As body weight and DR are complex traits influenced by numerous environmental and genetic factors, further genetic studies may be warranted to investigate the different effects of BMI on the risk of developing DR in Asian versus Western populations.

\section{Acknowledgements \\ Authors acknowledge Ms. Riswana Banu Binte Mohamed Abdul Sokor for providing with the administrative support. The current study was supported by the Singapore Ministry of Health's National Medical Research Council (NMRC), NMRC/1249/2010 (SiMES-2) and NMRC/CIRG/1371/2013 (SINDI-2). The funding source had no role in the design and conduct of the study; collection, management, analysis, and interpretation of the data; preparation, review, or approval of the manuscript; and decision to submit the manuscript for publication.}

\section{Author details}

'Singapore Eye Research Institute, Singapore National Eye Centre, The Academia, 20 College Road, Discovery Tower Level 6, Singapore 169856, Singapore. ${ }^{2}$ Department of Ophthalmology, Yong Loo Lin School of Medicine, National University of Singapore, Singapore, Singapore. ${ }^{3}$ Ophthalmology and Visual Sciences Academic Clinical Program, Duke-NUS Medical School, Singapore, Singapore. ${ }^{4}$ Centre for Quantitative Medicine, Duke-NUS Medical School, Singapore, Singapore

\section{Conflict of interest}

The authors declare that they have no conflict of interest.

\section{Publisher's note}

Springer Nature remains neutral with regard to jurisdictional claims in published maps and institutional affiliations.

Received: 18 September 2017 Revised: 8 January 2018 Accepted: 12 January 2018

Published online: 07 March 2018

\section{References}

1. Leasher, J. L. et al. Global estimates on the number of people blind or visually impaired by diabetic retinopathy: a meta-analysis from 1990 to 2010. Diabetes Care 39, 1643-1649 (2016).

2. Wong, T. Y., Cheung, C. M., Larsen, M., Sharma, S. \& Simo, R. Diabetic retinopathy. Nat. Rev. Dis. Prim. 2, 16012 (2016).

3. Cheung, N., Mitchell, P. \& Wong, T. Y. Diabetic retinopathy. Lancet 376, 124-136 (2010).

4. Ding, J. \& Wong, T. Y. Current epidemiology of diabetic retinopathy and diabetic macular edema. Curr. Diab Rep. 12, 346-354 (2012).

5. Wong, T. Y. et al. Relation between fasting glucose and retinopathy for diagnosis of diabetes: three population-based cross-sectional studies. Lancet 371, 736-743 (2008)

6. Yau, J. W. et al. Global prevalence and major risk factors of diabetic retinopathy. Diabetes Care 35, 556-564 (2012).

7. Nguyen, N. T., Nguyen, X. M., Lane, J. \& Wang, P. Relationship between obesity and diabetes in a US adult population: findings from the National Health and Nutrition Examination Survey, 1999-2006. Obes. Surg. 21, 351-355 (2011).

8. Dirani, M. et al. Are obesity and anthropometry risk factors for diabetic retinopathy? The diabetes management project. Invest Ophthalmol. Vis. Sci. 52, 4416-4421 (2011).

9. Consultation WHOE. Appropriate body-mass index for Asian populations and its implications for policy and intervention strategies. Lancet 363, 157-163 (2004).

10. Deurenberg-Yap, M., Chew, S. K. \& Deurenberg, P. Elevated body fat percentage and cardiovascular risks at low body mass index levels among Singaporean Chinese, Malays and Indians. Obes. Rev. 3, 209-215 (2002).

11. Rush, E. C., Freitas, I. \& Plank, L. D. Body size, body composition and fat distribution: comparative analysis of European, Maori, Pacific Island and Asian Indian adults. Br. J. Nutr. 102, 632-641 (2009). 
12. Ballard, D. J. et al. Risk factors for diabetic retinopathy: a population-based study in Rochester, Minnesota. Diabetes Care 9, 334-342 (1986).

13. Dowse, G. K. et al. Prevalence and risk factors for diabetic retinopathy in the multiethnic population of Mauritius. Am. J. Epidemiol. 147, 448-457 (1998).

14. Lim, L. S. et al. C-reactive protein, body mass index, and diabetic retinopathy. Invest Ophthalmol. Vis. Sci. 51, 4458-4463 (2010).

15. Man, R. E. et al. Differential association of generalized and abdominal obesity with diabetic retinopathy in Asian patients with type 2 diabetes. JAMA Ophthalmol. 134, 251-257 (2016)

16. Rema, M. et al. Prevalence of diabetic retinopathy in urban India: the Chennai Urban Rural Epidemiology Study (CURES) eye study, I. Invest Ophthalmol. Vis. Sci. 46, 2328-2333 (2005)

17. Rooney, D. et al. Body mass index and retinopathy in Asian populations with diabetes mellitus. Acta Diabetol. 52, 73-80 (2015).

18. van Leiden, H. A. et al. Risk factors for incident retinopathy in a diabetic and nondiabetic population: the Hoorn study. Arch. Ophthalmol. 121, 245-251 (2003).

19. $\mathrm{Xu}, \mathrm{J}$. et al. Prevalence and risk factors for diabetic retinopathy: the Beijing Communities Diabetes Study 6. Retina 32, 322-329 (2012).

20. Zhang, L., Krzentowski, G., Albert, A. \& Lefebvre, P. J. Risk of developing reti nopathy in diabetes control and complications trial type 1 diabetic patients with good or poor metabolic control. Diabetes Care. 24, 1275-1279 (2001).

21. Foong, A. W. et al. Rationale and methodology for a population-based study of eye diseases in Malay people: The Singapore Malay eye study (SiMES). Ophthalmic Epidemiol. 14, 25-35 (2007).

22. Lavanya, R. et al. Methodology of the Singapore Indian Chinese Cohort (SICC) eye study: quantifying ethnic variations in the epidemiology of eye diseases in Asians. Ophthalmic Epidemiol. 16, 325-336 (2009).

23. Cheung, C. M. G. et al. Six-year incidence of age-related macular degeneration in Asian Malays: The Singapore Malay Eye Study. Ophthalmology 124, 1305-1313 (2017)

24. Sabanayagam, C. et al. Singapore Indian Eye Study 2: methodology and impact of migration on systemic and eye outcomes. Clin. Exp. Ophthalmol. 45 , 779-789 (2017)

25. Kramer, $\mathrm{H}$. et al. Racial/ethnic differences in hypertension and hypertension treatment and control in the multi-ethnic study of atherosclerosis (MESA). Am. J. Hypertens. 17, 963-970 (2004)

26. Rosman, M. et al. Singapore Malay Eye Study: rationale and methodology of 6year follow-up study (SiMES-2). Clin. Exp. Ophthalmol. 40, 557-568 (2012).

27. Wong, T. Y. et al. Prevalence and risk factors for diabetic retinopathy: the Singapore Malay Eye Study. Ophthalmology 115, 1869-1875 (2008).

28. Group ETDRSR. Grading diabetic retinopathy from stereoscopic color fundus photographs--an extension of the modified Airlie House classification. ETDRS report number 10. Early Treatment Diabetic Retinopathy Study Research Group. Ophthalmology 98(5 Suppl), 786-806 (1991).
29. Zou, G. A modified poisson regression approach to prospective studies with binary data. Am. J. Epidemiol. 159, 702-706 (2004)

30. Narayan, K. M., Boyle, J. P., Thompson, T. J., Gregg, E. W. \& Williamson, D. F. Effect of BMl on lifetime risk for diabetes in the U.S. Diabetes Care 30 1562-1566 (2007).

31. Eckel, R. H. et al. Obesity and type 2 diabetes: what can be unified and what needs to be individualized? J. Clin. Endocrinol. Metab. 96, 1654-1663 (2011).

32. Deng, Y. \& Scherer, P. E. Adipokines as novel biomarkers and regulators of the metabolic syndrome. Ann. N. Y. Acad. Sci. 1212, E1-E19 (2010).

33. Larson-Meyer, D. E. et al. Intrahepatic and intramyocellular lipids are determinants of insulin resistance in prepubertal children. Diabetologia 54, 869-875 (2011).

34. Hotamisligil, G. S. \& Erbay, E. Nutrient sensing and inflammation in metabolic diseases. Nat. Rev. Immunol. 8, 923-934 (2008).

35. Weisberg, S. P. et al. Obesity is associated with macrophage accumulation in adipose tissue. J. Clin. Invest. 112, 1796-1808 (2003).

36. Deurenberg, P., Deurenberg-Yap, M. \& Guricci, S. Asians are different from Caucasians and from each other in their body mass index/body fat per cent relationship. Obes. Rev. 3, 141-146 (2002).

37. Beddhu, S., Pappas, L. M., Ramkumar, N. \& Samore, M. Effects of body size and body composition on survival in hemodialysis patients. J. Am. Soc. Nephrol. 14, 2366-2372 (2003)

38. Li, W. et al. Body mass index and stroke risk among patients with type 2 diabetes mellitus. Stroke 46, 164-169 (2015).

39. Oreopoulos, A. et al. Body mass index and mortality in heart failure: a metaanalysis. Am. Heart J. 156, 13-22 (2008)

40. Romero-Corral, A. et al. Association of bodyweight with total mortality and with cardiovascular events in coronary artery disease: a systematic review of cohort studies. Lancet 368, 666-678 (2006).

41. Gupta, D., Krueger, C. B. \& Lastra, G. Over-nutrition, obesity and insulin resistance in the development of beta-cell dysfunction. Curr. Diabetes Rev. 8, 76-83 (2012).

42. Klein, R., Klein, B. E., Moss, S. E., Davis, M. D. \& DeMets, D. L. The Wisconsin epidemiologic study of diabetic retinopathy. III. Prevalence and risk of diabetic retinopathy when age at diagnosis is 30 or more years. Arch. Ophthalmol. 102, 527-532 (1984)

43. Ozmen, B. \& Boyvada, S. The relationship between self-monitoring of blood glucose control and glycosylated haemoglobin in patients with type 2 diabetes with and without diabetic retinopathy. J. Diabetes Complicat. 17, 128-134 (2003).

44. Calle, E. E., Rodriguez, C., Walker-Thurmond, K. \& Thun, M. J. Overweight, obesity, and mortality from cancer in a prospectively studied cohort of U.S. adults. N. Engl. J. Med. 348, 1625-1638 (2003). 